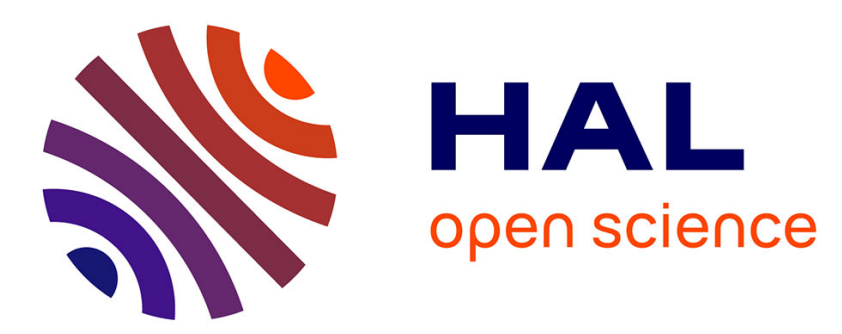

\title{
On The Optimal Number of Reflecting Elements for Reconfigurable Intelligent Surfaces
}

\author{
Alessio Zappone, Marco Di Renzo, Xiaojun Xi, Mérouane Debbah
}

\section{To cite this version:}

Alessio Zappone, Marco Di Renzo, Xiaojun Xi, Mérouane Debbah. On The Optimal Number of Reflecting Elements for Reconfigurable Intelligent Surfaces. IEEE Wireless Communications Letters, In press, 10.1109/LWC.2020.3034686 . hal-03020443

\section{HAL Id: hal-03020443 https://hal.science/hal-03020443}

Submitted on 23 Nov 2020

HAL is a multi-disciplinary open access archive for the deposit and dissemination of scientific research documents, whether they are published or not. The documents may come from teaching and research institutions in France or abroad, or from public or private research centers.
L'archive ouverte pluridisciplinaire HAL, est destinée au dépôt et à la diffusion de documents scientifiques de niveau recherche, publiés ou non, émanant des établissements d'enseignement et de recherche français ou étrangers, des laboratoires publics ou privés. 


\title{
On The Optimal Number of Reflecting Elements for Reconfigurable Intelligent Surfaces
}

\author{
Alessio Zappone, Senior Member IEEE, Marco Di Renzo, Fellow IEEE, Xiaojun Xi, Student Member, IEEE, \\ Merouane Debbah, Fellow IEEE
}

\begin{abstract}
This work considers a point-to-point link where a reconfigurable intelligent surface assists the communication between a transmitter and a receiver. The system rate, energy efficiency, and their trade-off are optimized by tuning the number of reflecting elements to be activated and the phase shifts that they apply. Unlike most previous works, the considered resource allocation problem explicitly accounts, at the design stage, for the time and energy that are necessary for channel estimation and for reporting the optimal configuration of the phase shifts to the reconfigurable intelligent surface. Numerical results confirm the optimality of the proposed methods and show the potential gains of reconfigurable intelligent surfaces.
\end{abstract}

\section{INTRODUCTION}

Recently, the concept of smart radio environment has emerged as a candidate architecture for future sixth generation (6G) networks [1], [2], [3], [4], [5], [6], wherein reconfigurable intelligent surfaces (RISs) are used to coat environmental objects that are present in the propagation environment. An RIS is a planar structure made of several individually tunable elements, called meta-atoms or passive scatterers, that can be programmed and appropriately reconfigured to control the phase of the incoming electromagnetic signal, by reflecting or refracting it towards specified locations [1]. In [7] and [8] the signal-to-noise ratio (SNR) scaling law of RIS-aided transmission is characterized, and the impact of hardware impairments is analyzed, respectively. Experimental testbeds have confirmed the potential gains of RISs [9], [10], [11], [12].

In the context of radio resource allocation, several works have recently appeared. In [13], the rate and energy efficiency (EE) of an RIS-based multiple-input single-output (MISO) downlink system are optimized by means of alternating optimization, fractional programming, and sequential optimization. A similar system setup is addressed in [14], and the problem of power minimization subject to minimum rate constraints is tackled by alternating optimization. In [15] and [16], sum-rate maximization in a MISO downlink system with orthogonal frequency division multiplexing is studied by optimizing the transmit beamformer and the RIS phase shifts with the aid of alternating optimization. An RIS with discrete

A. Zappone is with the University of Cassino and Southern Lazio, Cassino, Italy (alessio.zappone@unicas.it). M. Di Renzo and X. Xi are with Université Paris-Saclay, CNRS and CentraleSupélec, Laboratoire des Signaux et Systèmes, 91192 Gif-sur-Yvette, France (marco.direnzo@centralesupelec.fr), M. Debbah is with Huawei France R\&D, Boulogne-Billancourt, France (merouane.debbah@huawei.com).

M. Di Renzo's work was supported in part by the European Commission through the H2020 ARIADNE project under grant agreement 675806. phase shifts is considered in [17], wherein sum-rate maximization in a multi-user MISO system is addressed. In [18], a multi-user MISO channel is considered, in which an RIS is used to perform over-the-air computations. Therein, alternating optimization and difference convex programming are used for system optimization. In [19], multiple RISs are used in a massive multiple-input multiple-output (MIMO) setup, and the problem of maximizing the minimum of the users' signalto-interference-plus-noise-ratio with respect to the transmit precoder and the RISs phase shifts is considered. In [20], the problem of power control for physical-layer broadcasting under quality of service constraints for the mobile users is addressed. The downlink of a MIMO multi-cell system is considered in [21], wherein the problem of weighted sum-rate maximization is tackled by alternatively optimizing the base station beamformer and the RIS phase shifts. An RIS-based MISO millimiter-wave system is studied in [22], wherein the transmit beamforming and the phase shifts of multiple RISs are optimized. In [23], channel estimation and sum-rate maximization are investigated for a single-user uplink RIS-based system, by considering an RIS with discrete phase shifts. In [24], the sum-rate of a MIMO RIS-based system employing simultaneous wireless information and power transfer is maximized with respect to the transmitter beamforming and the RIS phase shifts. Power control for physical layer broadcasting in an RISbased network is investigated in [25]. Rate maximization in RIS-based indoor millimeter-waves systems is addressed in [26] by adjusting the RIS and the transmitter phase shifts.

The above literature survey shows that most works focus on how to maximize the rate of RIS-based systems with respect to the transmit power/beamforming and the RIS phase shifts, whereas the number of tunable elements at the RIS is not optimized. Moreover, previous works consider only the data transmission phase, while neglecting the overhead for channel estimation and for reporting the optimized configuration to the RIS. In contrast to these research works, this paper addresses the problem of jointly optimizing the RIS phase shifts and the number of tunable elements to be activated at the RIS, in order to optimize the rate, the EE and their trade-off. Notably, this is performed based on the model recently proposed in [27], which quantifies the impact of channel estimation and resource allocation feedback on the rate and EE of RIS-based systems.

The rest of the paper is organized as follows. Section II describes the system model, Sections III, IV, and V develop the proposed algorithm for the optimization of the rate, EE, and their trade-off, respectively. Section VI provides a numerical performance analysis, while Section VII concludes the work. 


\section{System Model And Problem Statement}

We consider a point-to-point system wherein a singleantenna transmitter and a single-antenna receiver communicate through an RIS equipped with $N$ individually tunable elements. This scenario models, for example, a device-to-device communication link, or a cellular network in which the base stations employ antenna selection to serve each user by a different antenna, and multi-user interference is suppressed through any orthogonal signaling technique. Also, [27] numerically shows that RISs are especially useful when few antennas are employed, since RIS optimization can compensate for the lack of multiple transmit and/or receive antennas.

We assume that the direct path between the transmitter and the receiver is not available, and denote by $\boldsymbol{h}=\left\{h_{n}\right\}_{n=1}^{N}$ and $\boldsymbol{g}=\left\{g_{n}\right\}_{n=1}^{N}$ the fast-fading channel vectors from the transmitter to the RIS and from the RIS to the receiver, respectively, and by $\delta$ the overall propagation path-loss. Before data communication can start, channel state information must be acquired. This can be accomplished by means of pilotbased methods which estimate the product channels $h_{n} g_{n}^{*}$, for all $n=1, \ldots, N$, with $*$ denoting complex conjugate. The specific estimation algorithm is inessential for the purpose of this work. We only make the baseline assumption that the product channels are estimated sequentially. Based on the estimated channels, the RIS configuration can be optimized and deployed. Specifically, the optimized RIS configuration must be communicated to the RIS. This is done by sending a configuration signal, which is processed at the RIS by a controller with minimal signal processing, transmission/reception, and power storage capabilities. The controller is a key component of RIS, which enables their reconfiguration capability in order to dynamically adapt to changes of the propagation channels [1, Fig. 4]. However, transmitting the control signal introduces a non-negligible overhead to the communication phase, especially for large $N$, since $N b_{F}$ bits must be encoded in the configuration signal, with $b_{F}$ the number of bits used to represent the phase applied by each RIS element. Denoting by $T$ the total duration of the time slot comprising the channel estimation phase of duration $T_{E}$, the control feedback phase of duration $T_{F}$, and the data communication phase of duration $T-T_{E}-T_{F}$, and defining $\beta=p \delta /\left(B N_{0}\right)$, with $B$ the communication bandwidth, $N_{0}$ the receive power spectral density, and $p$ the transmit power, the system rate and $\mathrm{EE}$ are expressed as

$$
\begin{aligned}
R(N) & =\left(1-\frac{T_{E}+T_{F}}{T}\right) B \log \left(1+\beta\left|\boldsymbol{g}^{H} \mathbf{\Phi} \boldsymbol{h}\right|^{2}\right) \\
\mathrm{EE}(N) & =R(N) / P_{t o t}
\end{aligned}
$$

where $\boldsymbol{\Phi}=\operatorname{diag}\left(e^{j \phi_{1}}, \ldots, e^{j \phi_{N}}\right)$ is the RIS phase matrix and $P_{t o t}$ is the total power consumption in the whole timeframe $T$. Following the model developed in [27], $T_{E}=T_{0}(N+1)$, with $T_{0}$ the duration of each pilot tone, and

$$
\begin{aligned}
T_{F} & =\frac{N b_{F}}{B_{F} \log \left(1+p_{F}\left|h_{F}\right|^{2} /\left(N_{0} B_{F}\right)\right)} \\
P_{t o t} & =P_{E}+\left(1-T_{E} / T\right) \mu p+T_{F} / T\left(\mu_{F} p_{F}-\mu p\right)+N P_{c, n}+P_{c, 0},
\end{aligned}
$$

since a power $p$ is used for $T-T_{E}-T_{F}$ seconds, with transmit amplifier efficiency $1 / \mu$, a power $p_{F}$ is used for $T_{F}$ seconds, with transmit amplifier efficiency $1 / \mu_{F}$, while hardware static power is consumed during the whole interval $T$, where $P_{c, n}$ is the hardware power required for each RIS element, $P_{c, 0}$ is the hardware power for all the other system components, and $P_{E}=T_{E} P_{0} / T$ is the power consumption during the channel estimation phase, with $P_{0}$ the power of each pilot tone.

The aim of this work is to optimize the RIS phase shift matrix $\boldsymbol{\Phi}$ and the number of tunable elements $N$ at the RIS, in order to optimize the system rate and EE in (1), and their trade-off. Note that the approach in this work differs from robust resource allocation methods which assume imperfect channel state information [28], [29], [30]. Indeed, we assume that reliable channel estimation is performed and the resulting overhead is appropriately accounted for in our system model.

\section{A. Optimization of $\boldsymbol{\Phi}$ and Upper-Bound of $N$}

Since $\boldsymbol{\Phi}$ does not affect the power consumption $P_{t o t}$, the optimal $\Phi$ for both the rate and the EE is obtained by maximizing the term $\left|\boldsymbol{g}^{H} \boldsymbol{\Phi} \boldsymbol{h}\right|^{2}$. This is accomplished by setting $\phi_{n}=-\angle g_{n}^{*} h_{n}$ for any $n$. With this choice, the received power at the destination is $p \delta\left(\sum_{n=1}^{N} \alpha_{n}\right)^{2}$, with $\alpha_{n}=\left|h_{n} g_{n}\right|$. On the other hand, since the received power can not be larger than the transmit power, it must hold that $\delta\left(\sum_{n=1}^{N} \alpha_{n}\right)^{2} \leqslant 1$. Defining $\alpha_{\max }=\max _{n} \alpha_{n}$, a sufficient condition for this to hold is $N \alpha_{\max } \sqrt{\delta} \leqslant 1$, which provides a simple upperbound on the maximum number of elements that can be placed on the RIS in order for the considered path-loss model to be physically meaningful. In addition, in order to prove the mathematical results to follow, we assume $\beta \alpha_{\max }^{2} \geqslant 1$. This is considered a practical assumption, since $\beta \alpha_{\text {max }}^{2}$ is the receive SNR over the reflection path with the largest gain. Thus, a wireless system in which $\beta \alpha_{\max }^{2}<1$, would have little practical value, because it could not provide the data-rate and reliability that are necessary in modern wireless systems. Since $N \alpha_{\max } \sqrt{\delta} \leqslant 1$, in order to enforce $\beta \alpha_{\max }^{2} \geqslant 1$, we must have $N^{2} \leqslant \beta / \delta$, which finally allows us to bound the maximum number $N_{\max }$ of elements at the RIS as

$$
N_{\max } \leqslant \min \left\{\left(\alpha_{\max } \sqrt{\delta}\right)^{-1}, \sqrt{\beta / \delta}\right\} .
$$

\section{RATE OPTIMIZATION}

Using the optimal $\mathbf{\Phi}$, the rate maximization problem is

$$
\max _{1 \leqslant N \leqslant \min \left\{N_{\max },[c / d]\right\}}(c-d N) B \log \left(1+\beta\left(\sum_{n=1}^{N} \alpha_{n}\right)^{2}\right)
$$

with $c=1-\frac{T_{0}}{T}, d=\frac{T_{0}}{T}+\frac{b_{F}}{T B_{F} \log \left(1+\frac{p_{F}\left|h_{F}\right|^{2}}{B_{F} N_{0}}\right)}$, and where, without loss of generality, we consider that the coefficients $\alpha_{n}$ are arranged in decreasing order of magnitude, i.e. $\alpha_{n} \geqslant \alpha_{n+1}$ for all $n=1, \ldots, \min \left\{N_{\max },\left\lfloor\frac{c}{d}\right\rfloor\right\}$, which also means that $\alpha_{\max }=\alpha_{1}$. The constraint in (5) ensures that the sum of the durations of the channel estimation and feedback phases is shorter than the total length of the frame, and that $N$ is smaller than its maximum feasible number $N_{\max }$. The challenge in solving (5) lies in the fact that the first factor of the objective 
decreases with $N$, while the second factor increases with $N$, which makes it difficult to determine the behavior of the rate $R$ as a function of the discrete variable $N$. In order to globally solve (5), the following result is instrumental.

Proposition 1: $R(N)$ in (5) is a unimodal function, i.e. it is either increasing with $N$ or, if there exists an $\bar{N}$ such that $R(\bar{N}) \geqslant R(\bar{N}+1), R(N)$ is decreasing for $N \geqslant \bar{N}$.

Proof: If $\bar{N}$ does not exist, the rate is increasing with $N$. If $\bar{N}$ exists, then defining $f(N)=B \log \left(1+\beta\left(\sum_{n=1}^{N} \alpha_{n}\right)^{2}\right)$, the condition $R(\bar{N}) \geqslant R(\bar{N}+1)$ is equivalent to

$$
(c-d \bar{N})(f(\bar{N}+1)-f(\bar{N})) \leqslant d f(\bar{N}+1) .
$$

Thus, the result holds if we can show that (6) implies that $R(\bar{N}+1) \geqslant R(\bar{N}+2)$, i.e.

$$
(c-d(\bar{N}+1))(f(\bar{N}+2)-f(\bar{N}+1)) \leqslant d f(\bar{N}+2) .
$$

At this point, let us show that, for any $N$, it holds

$$
f(N+1)-f(N) \geqslant f(N+2)-f(N+1) .
$$

To see this, expanding the square in $f(N+1)$ leads to

$$
\begin{aligned}
& f(N+1)-f(N)= \\
& B \log \left(1+\frac{\beta\left(\left(\sum_{n=1}^{N} \alpha_{n}\right)^{2}+\alpha_{N+1}^{2}+2 \alpha_{N+1} \sum_{n=1}^{N} \alpha_{n}\right)}{1+\beta\left(\sum_{n=1}^{N} \alpha_{n}\right)^{2}}\right)= \\
& B \log \left(1+\frac{\beta \alpha_{N+1}\left(\alpha_{N+1}+2 \sum_{n=1}^{N} \alpha_{n}\right)}{1+\beta\left(\sum_{n=1}^{N} \alpha_{n}\right)^{2}}\right)
\end{aligned}
$$

Similarly, it holds

$f(N+2)-f(N+1)=B \log \left(1+\frac{\beta \alpha_{N+2}\left(\alpha_{N+2}+2 \sum_{n=1}^{N+1} \alpha_{n}\right)}{1+\beta\left(\sum_{n=1}^{N+1} \alpha_{n}\right)^{2}}\right)$.

Then, the condition in (8) becomes

$$
\begin{gathered}
\frac{\alpha_{N+1}^{2}}{1+\beta\left(\sum_{n=1}^{N} \alpha_{n}\right)^{2}}+\frac{2 \alpha_{N+1} \sum_{n=1}^{N} \alpha_{n}}{1+\beta\left(\sum_{n=1}^{N} \alpha_{n}\right)^{2}} \geqslant \\
\frac{\alpha_{N+2}^{2}}{1+\beta\left(\sum_{n=1}^{N+1} \alpha_{n}\right)^{2}}+\frac{2 \alpha_{N+2} \sum_{n=1}^{N+1} \alpha_{n}}{1+\beta\left(\sum_{n=1}^{N+1} \alpha_{n}\right)^{2}} .
\end{gathered}
$$

Since $\alpha_{n} \geqslant \alpha_{n+1}$ for any $n=1, \ldots, N$, the first summand at the left-hand-side of (10) is larger than the first summand at the right-hand-side. Then, for (10) to hold it is sufficient that:

$$
\frac{\sum_{n=1}^{N} \alpha_{n}}{1+\beta\left(\sum_{n=1}^{N} \alpha_{n}\right)^{2}} \geqslant \frac{\sum_{n=1}^{N+1} \alpha_{n}}{1+\beta\left(\sum_{n=1}^{N+1} \alpha_{n}\right)^{2}} .
$$

Defining $z=\sum_{n=1}^{N} \alpha_{n}$, it can be seen that (11) is equivalent to showing that the function $g(z)=\frac{z}{1+\beta z^{2}}$ is decreasing. Computing the first-order derivative of $g(z)$, and setting it to be non-positive, yields $\beta \alpha_{1} \geqslant 1$, which holds on the feasible set of (5). Finally, exploiting (6), and observing that $f(N)$ is increasing in $N$ since each $\alpha_{n}$ is positive, it follows that

$$
\begin{aligned}
& (c-d(\bar{N}+1))(f(\bar{N}+2)-f(\bar{N}+1)) \leqslant \\
& (c-d \bar{N})(f(\bar{N}+1)-f(\bar{N})) \leqslant d f(\bar{N}+1) \leqslant d f(\bar{N}+2),
\end{aligned}
$$

and hence the proof follows.

Equipped with this result, Problem (5) can be solved by a greedy approach in which the tunable elements of the RIS are activated one at a time, following the decreasing order of magnitude of the coefficients $\left\{\alpha_{n}\right\}_{n}$, until a decrease in $R(N)$ is observed, or $N$ reaches its maximum allowed number. The procedure is stated in Algorithm 1, with $A(N)=R(N)$.

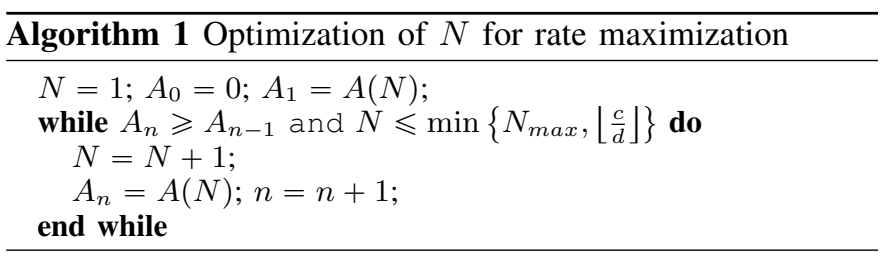

\section{ENERGY EFFICIENCY OPTIMIZATION}

Defining $\gamma=P_{c, 0}+P_{0} \frac{T_{0}}{T}+\mu p c$ and $\psi=\left(P_{0}-\mu p-1\right) \frac{T_{0}}{T}+$ $d+P_{c, n}$, the EE maximization problem is stated as

$$
\begin{aligned}
& \max _{N} \frac{(c-d N) f(N)}{\gamma+\psi N} \\
& \text { s.t. } 1 \leqslant N \leqslant \min \left\{N_{\max },\lfloor c / d]\right\} .
\end{aligned}
$$

Proposition 2 shows that the EE is unimodal, and thus (13a) can be globally solved by Algorithm 1 with $A(N)=\operatorname{EE}(N)$.

Proposition 2: $\mathrm{EE}(N)$ in (13a) is a unimodal function, i.e. it is either increasing with $N$ or, if there exists an $\bar{N}$ such that $\mathrm{EE}(\bar{N}) \geqslant \mathrm{EE}(\bar{N}+1), \mathrm{EE}(N)$ is decreasing for $N \geqslant \bar{N}$.

Proof: Proceeding as in the proof of Proposition 1, if $\bar{N}$ does not exist, then $\operatorname{EE}(N)$ is increasing with $N$. Instead, if $\bar{N}$ exists, the result follows if we can prove that $\operatorname{EE}(\bar{N}) \geqslant$ $\mathrm{EE}(\bar{N}+1)$ implies that $\operatorname{EE}(\bar{N}+1) \geqslant \operatorname{EE}(\bar{N}+2)$. First of all, let us observe that if $\bar{N}$ is such that $R(\bar{N}) \geqslant R(\bar{N}+1)$, then we already know from Proposition 1 that $\bar{N}$ falls in the range in which the rate function $R(N)$ is decreasing. This implies that the EE is decreasing for any $N \geqslant \bar{N}$, since increasing $N$ yields a lower numerator and a larger denominator. As a result, the non-trivial case to be considered is when $\bar{N}$ belongs to the range in which the rate is still increasing, i.e. the first decrease in the EE happens when the rate function is still increasing with $N$. Thus, without loss of generality, in the rest of this proof we assume $R(\bar{N}+2)>R(\bar{N}+1)>R(\bar{N})$. Next, let us observe that $\operatorname{EE}(\bar{N}) \geqslant \operatorname{EE}(\bar{N}+1)$ is equivalent to

$$
\bar{N} \leqslant-\frac{\gamma}{\psi}+\frac{R(\bar{N})}{R(\bar{N}+1)-R(\bar{N})} .
$$

Similarly, $\mathrm{EE}(\bar{N}+1) \geqslant \mathrm{EE}(\bar{N}+2)$ is equivalent to

$$
\bar{N} \leqslant-\frac{\gamma}{\psi}-1+\frac{R(\bar{N}+1)}{R(\bar{N}+2)-R(\bar{N}+1)} .
$$

At this point, we note that (14) can be written as

$$
\bar{N} \leqslant-\frac{\gamma}{\psi}-1+\frac{R(\bar{N}+1)}{R(\bar{N}+1)-R(\bar{N})},
$$


which implies (15) if we can show that $R(\bar{N}+2)-R(\bar{N}+1) \leqslant$ $R(\bar{N}+1)-R(\bar{N})$. To prove this, we observe that we have

$$
\begin{aligned}
& R(\bar{N}+2)-R(\bar{N}+1)= \\
& (c-d(\bar{N}+1))(f(\bar{N}+2)-f(\bar{N}+1))-d f(\bar{N}+2) \leqslant \\
& (c-d(\bar{N}+1))(f(\bar{N}+2)-f(\bar{N}+1))-d f(\bar{N}) \leqslant \\
& (c-d(\bar{N}+1))(f(\bar{N}+1)-f(\bar{N}))-d f(\bar{N})=R(\bar{N}+1)-R(\bar{N}),
\end{aligned}
$$

where the two equalities follow recalling that $R(N)=(c-$ $d N) f(N)$, while the two inequalities follow since $f(N)$ is non-negative, increasing, and such that $f(N+1)-f(N) \geqslant$ $f(N+2)-f(N+1)$, as proved in Proposition 1.

\section{RATE-EE MAXIMIZATION}

Rate-EE maximization is cast as the bi-objective problem

$$
\max _{N}\{R(N), \operatorname{EE}(N)\} \text {, s.t. } 1 \leqslant N \leqslant \min \left\{N_{\max },\lfloor c / d]\right\}
$$

By virtue of [31, Th. 3.4.5], all Pareto-optimal points of (18) can be obtained by solving

$$
\begin{aligned}
& \max _{N} \min \left\{w\left(R(N)-R_{o p t}\right),(1-w)\left(\mathrm{EE}(N)-\mathrm{EE}_{o p t}\right)\right\} \\
& \text { s.t. } 1 \leqslant N \leqslant \min \left\{N_{\text {max }},\left\lfloor\frac{c}{d}\right\rfloor\right\}
\end{aligned}
$$

for $w \in(0,1)$, with $R_{o p t}$ and $\mathrm{EE}_{\text {opt }}$ the individual maximizers of $R(N)$ and $\operatorname{EE}(N)$, as derived in Sections III and IV. The following proposition shows that $G(N)=\min \{w(R(N)-$ $\left.\left.R_{o p t}\right),(1-w)\left(\mathrm{EE}(N)-\mathrm{EE}_{o p t}\right)\right\}$ is unimodal, and thus Problem (19a) can be solved by Algorithm 1 with $A(N)=G(N)$.

Proposition 3: $G(N)$ is a unimodal function, i.e. it is either increasing with $N$, or, if there exists $\bar{N}$ such that $G(\bar{N}) \geqslant$ $G(\bar{N}+1), G(N)$ is decreasing for $N \geqslant \bar{N}$.

Proof: Propositions 1 and 2 ensure that $R(N)$ and $\operatorname{EE}(N)$ are unimodal functions, which implies that $G_{1}=w(R(N)-$ $\left.R_{\text {opt }}\right)$ and $G_{2}=(1-w)\left(\mathrm{EE}(N)-\mathrm{EE}_{\text {opt }}\right)$ are unimodal, too.

Let us consider first that both $G_{1}(N)$ and $G_{2}(N)$ have a finite maximizer, which we denote by $N_{1}$ and $N_{2}$, respectively. Without loss of generality, let us assume that $N_{1} \leqslant N_{2}$. Then, $G(N)$ is increasing for $N \leqslant N_{1}$ and decreasing for $N \geqslant N_{2}$. As for the range $N_{1}<N<N_{2}$, let us consider two cases:

(a) If $G_{1}\left(N_{1}\right) \leqslant G_{2}\left(N_{1}\right)$, then $G(N)=G_{1}(N)$ for $N_{1}<$ $N<N_{2}$, because in this range $G_{2}$ is increasing while $G_{1}$ is decreasing. As a result, $G(N)$ is increasing for $N \leqslant N_{1}$ and decreasing for $N>N_{1}$ and we have $\bar{N}=N_{1}$.

(b) If $G_{1}\left(N_{1}\right)>G_{2}\left(N_{1}\right)$, two cases can be considered: if $G_{1}(N) \geqslant G_{2}(N)$ for $N_{1} \leqslant N \leqslant N_{2}$, then $G(N)=G_{2}(N)$ for $N_{1} \leqslant N \leqslant N_{2}$ and the thesis holds with $\bar{N}=N_{2}$; if instead $G_{1}(N) \neq G_{2}(N)$ for $N_{1} \leqslant N \leqslant N_{2}$, then we can define $N_{3}$ as the smallest number such that $N_{1}<N_{3} \leqslant N_{2}$ and $G_{1}\left(N_{3}\right) \leqslant G_{2}\left(N_{3}\right)$. Thus, $G(N)=G_{2}(N)$ for $N_{1} \leqslant$ $N<N_{3}$, while $G(N)=G_{1}(N)$ for $N_{3} \leqslant N \leqslant N_{2}$. Then, it follows that $G(N)$ is increasing for $N<N_{3}$ and decreasing for $N \geqslant N_{3}$, and the thesis follows with $\bar{N}=N_{3}$.

Finally, we observe that the reasoning above includes as special cases the situations in which either $G_{1}$ or $G_{2}$ is monotonically increasing for all $N$, whereas if both $G_{1}$ and $G_{2}$ are monotonically increasing, then $G(N)$ is monotonically increasing and $\bar{N}$ does not exist.
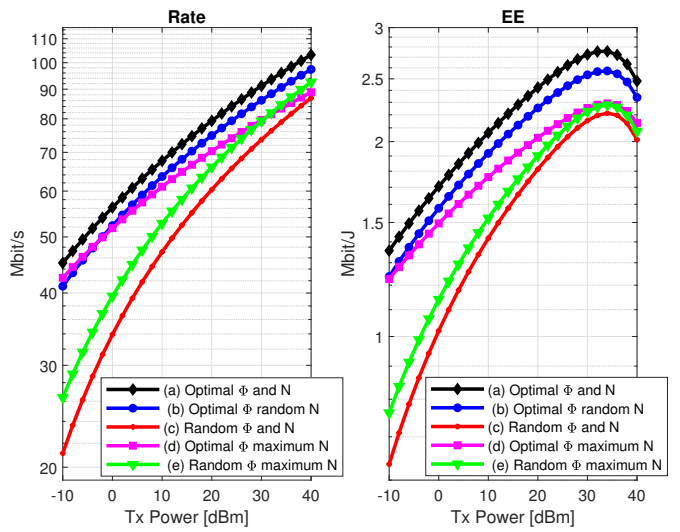

Fig. 1: Rate (left) and EE (right) versus $p$ for: (a) Optimal $N$ and $\mathbf{\Phi}$; (b) Random $N$ and optimal $\boldsymbol{\Phi}$; (c) Random $N$ and $\boldsymbol{\Phi}$; (d) $N=N_{\max }$ and optimal $\boldsymbol{\Phi}$; (e) $N=N_{\max }$ and random $\boldsymbol{\Phi}$

\section{NUMERICAL RESULTS}

In our numerical simulations we set $p_{F}=30 \mathrm{dBm}, B=$ $5 \mathrm{MHz}, B_{F}=1 \mathrm{MHz}, \delta=110 \mathrm{~dB}, N_{0}=-174 \mathrm{dBm} / \mathrm{Hz}$, $\mu=\mu_{F}=1, P_{c, 0}=45 \mathrm{dBm}, P_{c, n}=10 \mathrm{dBm}, b_{F}=16, T_{0}=$ $1 \mathrm{~ms}, P_{0}=10 \mathrm{dBm}$. Moreover, $h_{n} \sim \mathcal{C N}\left(v_{h}, 1\right)$ and $g_{n} \sim$ $\mathcal{C N}\left(v_{g}, 1\right)$. Thus, $\left|h_{n}\right|$ and $\left|g_{n}\right|$ are Rice distributed, where $v_{h}$ and $v_{g}$ are chosen so that the power of the line-of-sight path is four times larger than the power of all the other paths ${ }^{1}$. A similar model is used for the feedback channel $h_{F}$. Also, $N_{\max }=300$ and all presented results have been averaged over $10^{4}$ independent realizations of the channel vectors $\boldsymbol{h}$ and $\boldsymbol{g}$.

Fig. 1 shows the average rate and EE versus the transmit power $p$ achieved by the following schemes:

(a) Optimization of $N$ by Algorithm 1 and optimization of $\Phi$ as described in Section II-A.

(b) Random $N$ in $\left[1, N_{\max }\right]$ and optimization of $\boldsymbol{\Phi}$ as in Section II-A.

(c) Random $N$ in $\left[1, N_{\max }\right]$ and random $\phi_{n}$ in $[0,2 \pi]$ for all $n=1, \ldots, N$.

(d) $N=N_{\max }$ and optimization of $\boldsymbol{\Phi}$ as in Section II-A.

(e) $N=N_{\max }$ and random $\phi_{n}$ in $[0,2 \pi]$ for all $n=$ $1, \ldots, N$.

As for Schemes (c) and (e), we set $T_{F}=0$ and $T_{E}=T_{0}$, since no RIS optimization is performed. The results show that, accounting at the design stage for the overhead due to channel estimation and optimal RIS configuration outperforms the other schemes. In particular, Scheme (a) significantly outperforms Schemes (c) and (e), which employ suboptimal allocations of both $N$ and $\boldsymbol{\Phi}$, despite the fact that in these cases no overhead for RIS configuration is required. Thus, despite the overhead, an optimized RIS improves the performance.

Table I reports the average rate maximizer $N_{R}^{*}$ and the EE maximizer $N_{\mathrm{EE}}^{*}$ versus $p$, that correspond to the performance of Scheme (a) in Fig. 1. The results confirm that both the rate and the EE have a finite maximizer with $N$.

Finally, Fig. 2 shows the Rate-EE Pareto-frontier of the considered RIS-based system for $p=20 \mathrm{dBm}, p=30 \mathrm{dBm}$,

\footnotetext{
${ }^{1}$ In the numerical results, we did not enforce the condition $\beta \alpha_{\max } \geqslant 1$, which is only needed at the design stage.
} 


\begin{tabular}{|c|c|c|c|c|c|}
\hline & $0 \mathrm{dBm}$ & $10 \mathrm{dBm}$ & $20 \mathrm{dBm}$ & $30 \mathrm{dBm}$ & $40 \mathrm{dBm}$ \\
\hline$N_{R}^{*}$ & 198.89 & 197.32 & 193.38 & 184.74 & 172.04 \\
\hline$N_{\mathrm{EE}}^{*}$ & 187.05 & 167.29 & 147.01 & 133.92 & 145.99 \\
\hline
\end{tabular}

TABLE I: Average number of the RIS elements that maximize the rate and energy efficiency versus $p$

$p=40 \mathrm{dBm}$. For each value of $p$, the setups with $P_{c, n}=$ $10 \mathrm{dBm}$ and $P_{c, n}=15 \mathrm{dBm}$ are shown. As expected, a larger $P_{c, n}$ yields a wider Pareto-region, because the larger $P_{c, n}$ is, the more the rate and the EE are contrasting objectives.
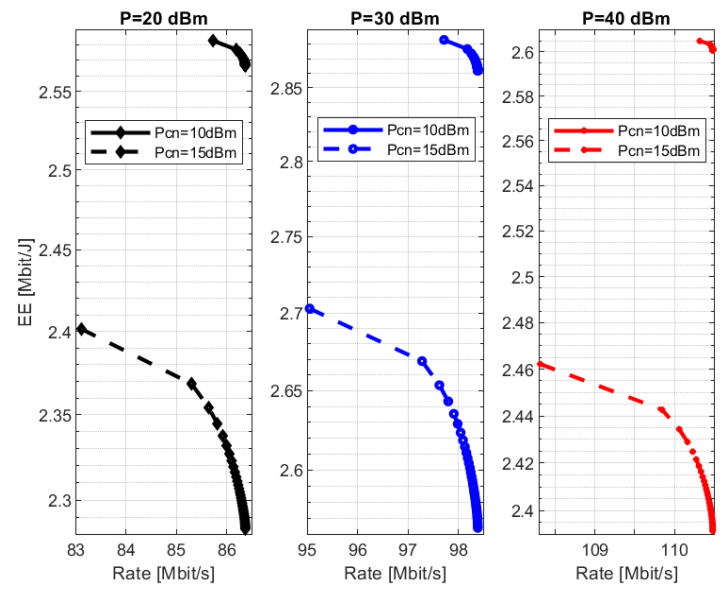

Fig. 2: Rate-EE Pareto region for $p=20 \mathrm{dBm}$ (left), $30 \mathrm{dBm}$ (middle), $40 \mathrm{dBm}$ (right), with $P_{c, n}=10 \mathrm{dBm}$ and $15 \mathrm{dBm}$

\section{CONCLUSIONS}

This work maximized the rate, EE, and their trade-off in a RIS-based system, as a function of the number and the phase shifts of the RIS elements, and by accounting for the overhead due to channel estimation and RIS configuration. Numerical results confirmed that overhead-aware resource allocation may significantly outperform sub-optimal methods. Future studies are needed to address more complex network topologies.

\section{REFERENCES}

[1] M. Di Renzo, A. Zappone, M. Debbah, M. Alouini, C. Yuen, J. de Rosny, and S. Tretyakov, "Smart radio environments empowered by reconfigurable intelligent surfaces: How it works, state of research, and road ahead," IEEE Journal on Selected Areas in Communications, 2020

[2] A. Zappone, M. Di Renzo, and M. Debbah, "Wireless networks design in the era of deep learning: Model-based, AI-based, or both?" IEEE Transactions on Communications, vol. 67, no. 10, pp. 7331-7376, 2019.

[3] M. Di Renzo et al., "Smart radio environments empowered by reconfigurable AI meta-surfaces: An idea whose time has come," EURASIP Journal on Wireless Communincations and Networking, vol. 129, 2019.

[4] C. Huang, S. Hu, G. C. Alexandropoulos, A. Zappone, C. Yuen, R. Zhang, M. Di Renzo, and M. Debbah, "Holographic MIMO surfaces for 6G wireless networks: Opportunities, challenges, and trends," IEEE Wireless Communications Magazine, vol. in press, 2020.

[5] E. Basar, M. Di Renzo, J. de Rosny, M. Debbah, M. S. Alouini, and R. Zhang, "Wireless communications through reconfigurable intelligent surfaces," IEEE Access, vol. 7, pp. 116753 - 116773, 2019.

[6] M. Di Renzo and et al., "Reconfigurable intelligent surfaces vs. relaying: Differences, similarities, and performance comparison," IEEE Open Journal of the Communications Society, vol. 1, pp. 798 - 807, 2019.

[7] X. Qian et al., "Beamforming through reconfigurable intelligent surfaces in single-user MIMO systems: SNR distribution and scaling laws in the presence of channel fading and phase noise," IEEE Wireless Communications Letters, in press, 2020.
[8] S. Zhou, W. Xu, K. Wang, M. Di Renzo, and M. Alouini, "Spectral and energy efficiency of IRS-assisted MISO communication with hardware impairments," IEEE Wireless Communications Letters, in press, 2020.

[9] W. Tang, M. Z. Chen, X. Chen, J. Y. Dai, Y. Han, M. Di Renzo, Y. Zeng, S. Jin, Q. Cheng, and T. J. Cui, "Wireless communications with reconfigurable intelligent surface: Path loss modeling and experimental measurement," IEEE Transactions on Wireless Communications, in press, 2020.

[10] W. Tang et al., "Wireless communications with programmable metasurface: Transceiver design and experimental results," China Communications, vol. 16, no. 5, pp. 46-61, May 2019.

[11] — "Programmable metasurface-based RF chain-free 8PSK wireless transmitter," IEEE Electronic Letters, vol. 55, no. 7, pp. 417-420, 2019.

[12] L. Dai et al., "Reconfigurable intelligent surface-based wireless communication: Antenna design, prototyping and experimental results," IEEE Access, vol. 8, pp. 45913 - 45923, 2020.

[13] C. Huang, A. Zappone, G. C. Alexandropoulos, M. Debbah, and C. Yuen, "Reconfigurable intelligent surfaces for energy efficiency in wireless communication," IEEE Trans. on Wireless Commun., vol. 18, no. 8, pp. 4157-4170, 2019.

[14] Q. Wu and R. Zhang, "Intelligent reflecting surface enhanced wireless network: Joint active and passive beamforming design," IEEE Transactions on Wireless Communications, vol. 18, no. 11, pp. 5394-5409, 2019.

[15] Y. Yang, S. Zhang, and R. Zhang, "IRS-enhanced OFDM: Power allocation and passive array optimization," IEEE Wireless Communications Letters, vol. 9, no. 6, pp. 760 - 764, 2020.

[16] X. Yu, D. Xu, and R. Schober, "MISO wireless communication systems via intelligent reflecting surfaces," in 2019 IEEE/CIC International Conference on Communications in China (ICCC), 2019.

[17] R. Liu, H. Li, M. Li, and Q. Liu, "Symbol-level precoding design for intelligent reflecting surface assisted multi-user MIMO systems,' https://arxiv.org/abs/1909.01015, 2019.

[18] T. Jiang and Y. Shi, "Over-the-air computation via intelligent reflecting surfaces," 2019 IEEE Global Communications Conference, 2019.

[19] X. Li, J. Fang, F. Gao, and H. Li, "Joint active and passive beamforming for intelligent reflecting surface-assisted massive MIMO systems," https://arxiv.org/abs/1912.00728, 2019.

[20] H. Han et al., "Intelligent reflecting surface aided network: Power control for physical-layer broadcasting," arxiv.org/abs/1910.14383, 2019.

[21] C. Pan et al., "Multicell MIMO communications relying on intelligent reflecting surface," IEEE Transactions on Wireless Communications, in press, 2020.

[22] P. Wang, J. Fang, X. Yuan, Z. D. Chen, H. Duan, and H. Li, "Intelligent reflecting surface-assisted millimeter wave communications: Joint active and passive precoding design," https://arxiv.org/abs/1908.10734, 2019.

[23] C. You, B. Zheng, and R. Zhang, "Intelligent reflecting surface with discrete phase shifts: Channel estimation and passive beamforming," IEEE Journal on Selected Areas in Communications, in press, 2020.

[24] C. Pan, H. Ren, K. Wang, M. Elkashlan, A. Nallanathan, J. Wang, and L. Hanzo, "Intelligent reflecting surface aided MIMO broadcasting for simultaneous wireless information and power transfer," IEEE Journal on Selected Areas in Communications, in press, 2020.

[25] H. Han, J. Zhao, Z. Xiong, D. Niyato, M. Di Renzo, Q. V. Pham, and W. Lu, "Intelligent reconfigurable surface aided power control for physical-layer broadcasting," arXiv preprint arXiv:1912.03468, 2020.

[26] N. S. Perović, M. Di Renzo, and M. F. Flanagan, "Channel capacity optimization using reconfigurable intelligent surfaces in indoor mmWave environments," 2020 International Communications Conference (ICC 2020), 2020.

[27] A. Zappone, M. Di Renzo, F. Shams, X. Qian, and M. Debbah, "Overhead-aware design of reconfigurable intelligent surfaces in smart radio environments," IEEE Transactions on Wireless Communications, in press, 2020.

[28] G. Zhou, C. Pan, H. Ren, K. Wang, and A. Nallanathan, "A framework of robust transmission design for IRS-aided MISO communications with imperfect cascaded channels," https://arxiv.org/abs/2001.07054, 2020.

[29] S. Hong, C. Pan, H. Ren, K. Wang, K. Chai, and A. Nallanathan, "Robust transmission design for intelligent reflecting surface aided secure communication systems with imperfect cascaded CSI," https://arxiv.org/abs/2004.11580, 2020.

[30] G. Zhou et al., "Robust beamforming design for intelligent reflecting surfaceaided MISO communication systems," IEEE Wireless Communications Letters, in press, 2020.

[31] K. Miettinen, Nonlinear Multiobjective Optimization. Springer, 1999. 